\title{
Early continuous ST segment monitoring in unstable angina: prognostic value additional to the clinical characteristics and the admission electrocardiogram
}

Deven J Patel, Diana R Holdright, Charles J Knight, David Mulcahy, Bharat Thakrar, Christine Wright, Jane Sparrow, Margaret Wicks, William Hubbard, Rod Thomas, George C Sutton, Gordon Hendry, Henry Purcell, Kim Fox

\begin{abstract}
Background and objective-In unstable angina, clinical characteristics, resting electrocardiography, and early continuous ST segment monitoring have been individually reported to identify subgroups at increased risk of adverse outcome. It is not known, however, whether continuous ST monitoring provides additional prognostic information in such a setting.
\end{abstract}

Design-Observational study of 212 patients with unstable angina without evidence of acute myocardial infarction admitted to district general hospitals, who had participated in a randomised study comparing heparin and aspirin treatment versus aspirin alone.

Methods-Clinical variables and a 12 lead electrocardiogram (ECG) were recorded at admission, and treatment was standardised to include aspirin, atenolol, diltiazem, and intravenous glyceryl trinitrate, in addition to intravenous heparin (randomised treatment). Continuous ST segment monitoring was performed for $48 \mathrm{~h}$ and all inhospital adverse events were recorded.

Results-The admission ECG was normal in 61 patients $(29 \%)$, showed ST depression in $59(28 \%)(17 \geqslant 0.1 \mathrm{mV})$, and $T$ wave changes in a further $69(33 \%)$. The remaining 23 had $Q$ waves (18), right bundle branch block (four), or ST elevation (one). During $8963 \mathrm{~h}$ of continuous ST segment monitoring (mean $42 \cdot 3$ h/patient), 132 episodes of transient myocardial ischaemia (104 silent) were recorded in 32 patients (15\%). Forty patients $(19 \%)$ had an adverse event (cardiac deaths $(n=3)$, non-fatal myocardial infarction $(n=6)$ and, emergency revascularisation $(n=31)$ ). Both admission ECG ST depression ( $P=0.02)$, and transient ischaemia $(P<0.001)$ predicted an increased risk of non-fatal myocardial infarction or death, while no patients with a normal ECG died or had a myocardial infarction. Adverse outcome was predicted by admission ECG ST depression (regardless of severity) (odds ratio (OR) 3.41) $(P<0.001)$, and maintenance $\beta$ blocker treatment (OR 2.95) $(P<0.01)$. A normal ECG predicted a favourable outcome (OR 0.38) $(P=0.04)$, while $T$ wave or other ECG changes were not predictive of outcome. Transient ischaemia was the strongest predictor of adverse prognosis (OR 4.61) (P< 0.001), retaining independent predictive value in multivariate analysis (OR 2.94) $(P=0.03)$, as did maintenance $\beta$ blocker treatment (OR 2.85) $(P=0.01)$ and admission ECG ST depression, which showed a trend towards independent predictive value (OR 2-11) (P= 0.076).

Conclusions-Patients with unstable angina and a normal admission ECG have a good prognosis, while ST segment depression predicts an adverse outcome. Transient myocardial ischaemia detected by continuous ST segment monitoring in such patients receiving optimal medical treatment provides prognostic information additional to that gleaned from the clinical characteristics or the admission ECG.

(Heart 1996;75:222-228)

Keywords: unstable angina; electrocardiography; prognosis; transient myocardial ischaemia

Although the clinical diagnosis of unstable angina encompasses a heterogeneous group of patients with differing underlying pathophysiological mechanisms, there is no doubt that it is associated with an adverse prognosis in the short term..$^{1-6}$ Even in the absence of confirmatory diagnostic tests, risk stratification can be performed based on the clinical characteristics together with analysis of the resting 12 lead electrocardiogram (ECG). The presence of significant $\mathrm{ST}$ segment depression $(\geqslant 0.1$ $\mathrm{mV}),{ }^{137-9}$ or anterior $\mathrm{T}$ wave inversion, ${ }^{10-12}$ has been shown to be associated with an increased risk of an unfavourable outcome.

Following the widespread use of ambulatory ST segment monitoring in stable coronary disease, continuous monitoring of the ST segment has also been utilised for risk stratification in unstable angina, ${ }^{713-17}$ and the presence of episodes of transient ST segment depression has been shown to predict adverse outcome. ${ }^{713-16}$ Some of these studies, however, preselected patients with ST segment depression of $\geqslant 0.1 \mathrm{mV}$ or $\mathrm{T}$ wave changes on a resting ECG. ${ }^{14} 15$ Conflicting results have been reported in two reports of unselected groups. ${ }^{717}$ 
Most of these earlier studies were performed before routine use of aspirin or heparin, and standardisation of antianginal treatment was not always attempted. Furthermore, it has not been established whether continuous ST segment monitoring provides any further prognostic information over and above the admission clinical characteristics and ECG. The prognostic significance of the resting 12 lead ECG and the findings of continuous ST segment monitoring may have been influenced by these factors, questioning their value in patients admitted with unstable angina.

We report on the prognostic value of the resting admission ECG and subsequent $48 \mathrm{~h}$ of continuous ST segment monitoring in patients with a clinical diagnosis of unstable angina treated with maximal antianginal treatment and oral aspirin, who participated in a randomised study assessing the efficacy of intravenous heparin and aspirin compared with that of aspirin alone.

\section{Patients and methods \\ PATIENTS}

The study data were collected as part of a randomised study evaluating the use of heparin in addition to aspirin and maximal antianginal treatment, which has been reported previously. ${ }^{18}$ Three district general hospitals were involved in the recruitment of patients between 1990 and 1992 and all results were collated and analysed at the Royal Brompton Hospital.

Patients were included if they were aged between 30 and 75 years, had presented within $24 \mathrm{~h}$ of an episode of typical anginal pain, and were willing to give informed consent. Patients presenting with new onset angina, sudden exacerbation of previously stable angina, or angina within one month of myocardial infarction were included. Symptoms had to have occurred at rest or with minimal effort and to be of sufficient severity to warrant emergency hospitalisation. Patients with prolonged chest pain, evidence of persistent ST elevation, or evolving $Q$ waves were excluded. Patients were also excluded if the resting ECG showed evidence of left ventricular hypertrophy with strain pattern, or left bundle branch block morphology, or they were receiving drugs that might influence interpretation of ST segment changes.

All patients were treated with oral aspirin $150 \mathrm{mg}$ daily and maximal antianginal treatment consisting of an intravenous infusion of nitrates, an oral $\beta$ blocker, and oral diltiazem $60 \mathrm{mg}$ thrice daily. Drug dosage was adjusted according to symptoms and haemodynamic response. Patients were then randomised to receive either intravenous heparin or no heparin with anticoagulation maintained to achieve an activated partial thromboplastin time ratio of $1 \cdot 5-2 \cdot 5$ of control value.

A total of 285 patients were recruited into the study. Seventy three patients, however, had cardiac enzymes that were significantly increased within the first two samples, or developed new $Q$ waves on serial ECGs con- firming the presence of myocardial infarction. These patients have been excluded from the analysis and the remaining 212 with unstable angina form the basis of this report.

The study was approved by the ethics committee of all participating centres and all patients gave informed written consent before enrolment, in accordance with the local ethics committee regulations.

RESTING ECG AND ST SEGMENT MONITORING A resting 12 lead ECG was recorded before entry to the study and was followed by $48 \mathrm{~h}$ of continuous ST segment monitoring using frequency modulated Oxford Medilog MR35 dual channel recorders with a frequency response of $0.05-40 \mathrm{~Hz}$. The admission ECG was analysed independently by two observers who were blinded to the subsequent outcome. Where there was discrepancy between the two observers the findings were decided by consensus or a third observer. ST segment depression, ST elevation, biphasic or inverted $\mathrm{T}$ waves, old $Q$ waves as well as any other abnormalities were noted. Neither observer recorded ST segment depression when it was $<0.02 \mathrm{mV}$.

The ST segment tapes were also analysed blind to outcome by visual review at $60-120$ times normal speed using the Oxford MA20 analyser. All potential episodes of ST segment depression were printed and measured. The automatic ST trend was also examined for any further possible episodes and these were confirmed by measurement of the printed ECG trace. Significant ST segment depression was defined as $\geqslant 0.1 \mathrm{mV}$ planar or downsloping ST segment depression, from baseline at 80 $\mathrm{ms}$ after the $\mathrm{J}$ point lasting for more than $60 \mathrm{~s}$. ST elevation was defined as the development of $\geqslant 0.2 \mathrm{mV}$ upward ST segment deviation at the J point. Each episode had to be separated by a return of the ST segment to baseline for at least one minute to be counted as a discrete episode. Changes in the $\mathrm{T}$ wave vector in the absence of ST segment changes were not recorded or included in the analysis. The frequency, duration of episodes, and their association with chest pain, based on a chest pain chart, were also noted.

\section{INHOSPITAL OUTCOME}

Inhospital outcome was documented for each patient with cause of death ascertained from clinical history and postmortem examination where available. Myocardial infarction was defined as the development of prolonged chest pain associated with either a rise in cardiac enzymes of at least two times the upper limit of normal, or the development of new $Q$ waves on serial ECG. Refractory unstable angina was defined as the need for emergency coronary angioplasty or bypass surgery for uncontrolled anginal symptoms and required emergency transfer of these patients from district general hospitals to cardiothoracic centres; the revascularisation procedure had to be performed within this inhospital phase to be included as an event. The recruiting centres were not informed of the results of the ST segment 
monitoring to prevent referral bias. A combined end point of unfavourable outcome was defined as the occurrence of cardiac death, non-fatal myocardial infarction, or emergency revascularisation.

\section{STATISTICAL ANALYSIS}

Analysis was performed using the BMDP statistical package. $\chi^{2}$ analysis was used to compare baseline categorical variables and the Mann-Whitney $U$ test was used to compare continuous but non-normally distributed data. Fisher's exact test was used for significance testing for the risk of death or myocardial infarction as the rate of these events was low. Two sided $P$ values of significance are quoted throughout and a $P$ value of $<0.05$ was considered to represent significance. Univariate logistic regression analysis for the combined end point of any adverse outcome was performed using all clinical variables, 12 lead ECG findings, and the presence of transient ischaemia with continuous ST segment monitoring. Forward stepwise logistic regression analysis was performed to predict unfavourable outcome using all variables of potential significance identified in the univariate analysis. A P value of $>0.4$ was used for removal of non-significant variables, and $P<0.2$ for entering variables of potential significance, in the model. Odds ratios (OR) are presented together with $95 \%$ confidence intervals $(\mathrm{CI})$ :

\section{Results}

CLINICAL CHARACTERISTICS

Table 1 shows the clinical characteristics of the study population $(n=212)$. Mean age of the study population was $58 \cdot 9$ (range $30-77$ ) years and almost $80 \%$ were men. A prior history of myocardial infarction was present in over $40 \%$, and $90(42.5 \%)$ of the recruited patients had new onset anginal symptoms. A definite diagnosis of coronary artery disease could be made in 124 patients $(58.5 \%)$ on the basis of documented previous infarction, $Q$ waves on the resting ECG, coronary angiographic findings, or the subsequent development of myocardial infarction or death. The angiography rate was low as the study was conducted in hospitals without on site angiographic facilities and this investigation was not required as part of the study protocol.

At the time of admission 81 patients $(38.2 \%)$ were already receiving aspirin and 81 $(38 \cdot 2 \%)$ were taking a $\beta$ blocker. Calcium

Table 1 Clinical characteristics and risk factor profile

\begin{tabular}{lc}
\hline Characteristic & $\begin{array}{c}\text { No of patients } \\
(n=212)\end{array}$ \\
\hline Mean (range) age (years) & $58 \cdot 9(30-77)$ \\
Men & $169(79 \cdot 7)$ \\
Smoking history & $175(82 \cdot 5)$ \\
Family history & $123(58 \cdot 0)$ \\
Diabetes & $14(6 \cdot 6)$ \\
Hypertension & $73(34 \cdot 4)$ \\
Previous stable angina & $121(57 \cdot 1)$ \\
Previous myocardial infarction & $91(42 \cdot 9)$ \\
New onset angina & $90(42 \cdot 5)$ \\
Unstable angina post-myocardial infarction & 1 \\
\hline
\end{tabular}

Values in parentheses are percentages.
Table 2 Admission resting 12 lead ECG findings

\begin{tabular}{ll}
\hline ECG findings & No of patients \\
\hline Normal & $61(28 \cdot 8)$ \\
Any degree of ST depression & $59(27 \cdot 8)$ \\
ST depression $\geqslant 0 \cdot 1 \mathrm{mV}$ & $17(8 \cdot 0)$ \\
ST depression $<0 \cdot 1 \mathrm{mV}$ & $42(19 \cdot 8)$ \\
Biphasic/inverted T wave & $95(44 \cdot 8)$ \\
Biphasic/inverted T wave without & $69(32 \cdot 5)$ \\
ST depression & $18(8 \cdot 5)$ \\
ST elevation & $47(22 \cdot 2)$ \\
Q wavest & $4(1 \cdot 9)$ \\
RBBB & \\
\hline
\end{tabular}

Values in parethesis are percentages.

${ }^{\star} \mathrm{ST}$ elevation was associated with $\mathrm{Q}$ waves in 12 patients, $T$ wave changes in four, ST depression in one and isolated in one. tQ waves were not associated with a rise in cardiac one. $+Q$ waves were not associated with a rise in cardiac
enzymes in the first two days and were present in the absence of ST segment or T wave changes in 18 patients $(8.5 \%)$.

ST segment or T wave changes in
$\mathrm{RBBB}$, right bundle branch block.

channel blockers were regular medication for 70 patients $(33.0 \%)$ and long acting nitrates for $80(37 \cdot 7 \%)$. Some 199 patients $(93.9 \%)$ received aspirin for the $48 \mathrm{~h}$ period and there was either poor tolerance, or a definite contraindication identified after recruitment in the remaining 13. Oral $\beta$ blockers were tolerated by 158 patients $(74.5 \%)$ and diltiazem by 163 $(76.9 \%)$; almost all patients $(97 \cdot 2 \%)$ were maintained on intravenous nitrates for the 48 $\mathrm{h}$ period. Intravenous heparin was administered to 109 patients $(51.4 \%)$ in accordance with the randomisation schedule.

RESTING 12 LEAD ECG

Table 2 shows the frequency of abnormalities on the resting ECG. Sixty one patients $(28 \cdot 8 \%)$ had a normal resting ECG and 59 $(27 \cdot 8 \%)$ ST segment depression, of whom 17 $(8.0 \%)$ had $\geqslant 0.1 \mathrm{mV} \mathrm{ST}$ depression and 42 $(19.8 \%)<0.1 \mathrm{mV}$ ST depression. ST elevation of $\geqslant 0.1 \mathrm{mV}$ was present on 18 ECGs, but this was associated with old $Q$ waves in 12 patients, $T$ wave changes in four, with marked ST depression in one, and was isolated in only one. Biphasic or inverted $\mathrm{T}$ waves were present in 95 patients $(44.8 \%)$, and in 69 $(32.5 \%)$ of these there was no associated depression of the ST segment. Pathological Q waves were present on $47(22 \cdot 2 \%)$ of the ECGs and were isolated from ST segment or $\mathrm{T}$ wave changes in $18(8 \cdot 5 \%)$.

\section{AMBULATORY MONITORING}

A total of $8963 \mathrm{~h}$ of ST segment monitoring were analysable (mean $42 \cdot 3 \mathrm{~h} /$ patient). Thirty two patients $(15 \cdot 1 \%)$ had a total of 132 episodes of ischaemia. In those with at least one episode the median frequency was $2 \cdot 5$ episodes/48 h. Some 104 episodes (78.8\%) of transient ischaemia were silent with 14 of the 32 patients having only silent ischaemia. The median (range) total duration of ischaemia over the recording period was $55(2-530) \mathrm{min}$ and nine patients had $\geqslant 60 \mathrm{~min} / 24 \mathrm{~h}$ of recording. Figure 1 shows the distribution of transient ischaemia according to the admission ECG changes; no episodes occurred in those with a normal admission ECG. Forty episodes $(30.3 \%)$ occurred in eight of 17 patients $(47 \cdot 1 \%)$ with $\geqslant 0.1 \mathrm{mV} \mathrm{ST}$ depression on the 12 lead ECG, and 65 episodes (49.2\%) in 13 of 42 patients $(31.0 \%)$ with $<0.1 \mathrm{mV}$ ST 
Figure 1 (A) Patients with at least one episode of transient myocardial ischaemia (TMI) according to admission ECG changes. (B) Proportion of the total number of TMI episodes. Patients with a normal admission ECG ( $n=61)$ did not subsequently have TMI during continuous ST monitoring, nor did the few patients with ST elevation or other ECG abnormalities. ST dep, $S T$ depression.

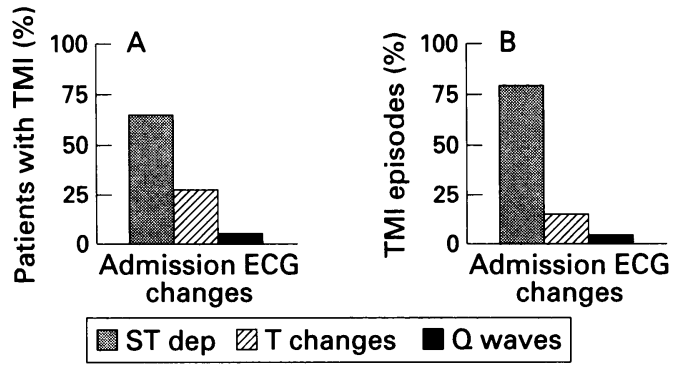

Table 3 Adverse events during the inhospital phase

\begin{tabular}{lc}
\hline Event & No of patients \\
\hline Cardiac death & $3(1 \cdot 4)$ \\
Non-fatal myocardial infarction & $6(2 \cdot 8)$ \\
Cardiac death or non-fatal myocardial & $9(4 \cdot 2)^{\star} \dagger$ \\
infarction & $14(6 \cdot 6)$ \\
Emergency CABG & $18(8 \cdot 5) \ddagger$ \\
Emergency PTCA & $32(15 \cdot 1)$ \\
Any emergency revascularisation & $40(18 \cdot 9)$ \\
Any adverse event &
\end{tabular}

Values in parentheses are percentages.

$\star P=0.015$ for ST segment depression on resting ECG. $+\mathrm{P}<$ 0.001 for transient myocardial ischaemia during continuous 0.001 for transient myocardial ischaemia during continuous ST segment monitoring. ‡One patient who developed myocardial infarction required emergency PTCA for refractory angina. PTCA, percutaneous transluminal coronary angioplasty; CABG, coronary artery bypass grafting.

depression. Only nine of 70 patients $(12 \cdot 9 \%)$ with isolated $\mathrm{T}$ wave changes had a total of 20 $(15 \cdot 2 \%)$ episodes of ischaemia, and two of 18 $(11 \cdot 1 \%)$ with isolated $Q$ waves had seven episodes $(5 \cdot 3 \%)$ of ischaemia.

Transient ischaemia was as common in those given maintenance $\beta$ blockers at admission $(n=81)(15(18.5 \%)$ having ischaemia with a median of 3 (range 1-15) episodes) as that in patients not receiving maintenance $\beta$ blockers $(\mathrm{n}=131) \quad(17 \quad(13.0 \%)$ having ischaemia with a median of 2 (range 1-11) episodes) $(\mathrm{P}=$ not significant $(\mathrm{NS}))$. The total duration of ischaemia (median (range)) was, however, significantly higher in those receiving maintenance $\beta$ blockers than those without such treatment $68(19-530) v 32(2-201) \mathrm{min}$ $(P=0.02)$. Patients receiving calcium antagonists at admission $(n=70)$ did not have greater ischaemia (13 (18.6\%) v 19 (13.4\%), $\mathrm{P}=\mathrm{NS})$, median episodes of ischaemia (2 (range 1-15) $v 3$ (range 1-12) episodes, $(\mathrm{P}=$ NS), or duration of ischaemia (median 45 (range 5-308) $v 61$ (range 2-530) $\mathrm{min}, \mathrm{P}=$ NS) than those without such medication. Patients given maintenance aspirin treatment $(n=81)$ also had a similar degree of ischaemia compared with that in those admitted without aspirin medication $(9(11 \cdot 1 \%) v 23(17 \cdot 6 \%)$ patients, $\mathrm{P}=\mathrm{NS}$; median episodes 2 (range 1-15) v 3 (range 1-12), $\mathrm{P}=\mathrm{NS}$; and median duration 61 (range 2-530) $v 45$ (range 5-308) $\min , \mathrm{P}=\mathrm{NS}$ ).

\section{OUTCOME}

Median duration of hospitalisation was 6 days.

Table 3 shows that there were three $(1 \cdot 4 \%)$ cardiac deaths, $6(2 \cdot 8 \%)$ non-fatal myocardial infarctions, and 32 emergency revascularisation procedures for refractory unstable angina. One patient who developed myocardial infarction underwent percutaneous transluminal coronary angioplasty for continuing chest pain, thus 31 (14.6\%) had emergency revascularisation as a primary event, giving an overall event rate of $18.9 \%$ ( 40 patients).

No clinical demographic variables were able to predict the occurrence of cardiac death or non-fatal myocardial infarction. Figure 2 shows the number of patients with adverse outcome (death, myocardial infarction, or any adverse event) according to the changes present on the admission ECG and the findings of ST segment monitoring. ST segment depression on the resting ECG was associated with a significantly greater frequency of myocardial infarction or death $(P=0.015)$ as was the presence of transient ST segment shift during ST segment monitoring $(P<0.001)$ with no events occurring in patients with a normal resting ECG at presentation $(P=0.06)$.

\section{COMBINED ADVERSE OUTCOME}

Of the clinical characteristics, univariate analysis (table 4) showed only the use of maintenance $\beta$ blockers before admission to be
Figure 2 Flow diagram showing the breakdown of patient numbers in relation to the admission ECG findings, the presence or absence of transient myocardial ischaemia (TMI), and subsequent adverse outcome. The occurrence of death and non-fatal myocardial infarction (MI) are shown separately to any adverse event which includes death, MI, and emergency revascularisation. ECG ST segment depression is separated into a group with minor ST depression $(<1$ $\mathrm{mm}$ ), and those with at least $1 \mathrm{~mm}$ depression. Other ECG changes include isolated $T$ wave changes, $S T$ segment elevation, and isolated $Q$ waves.

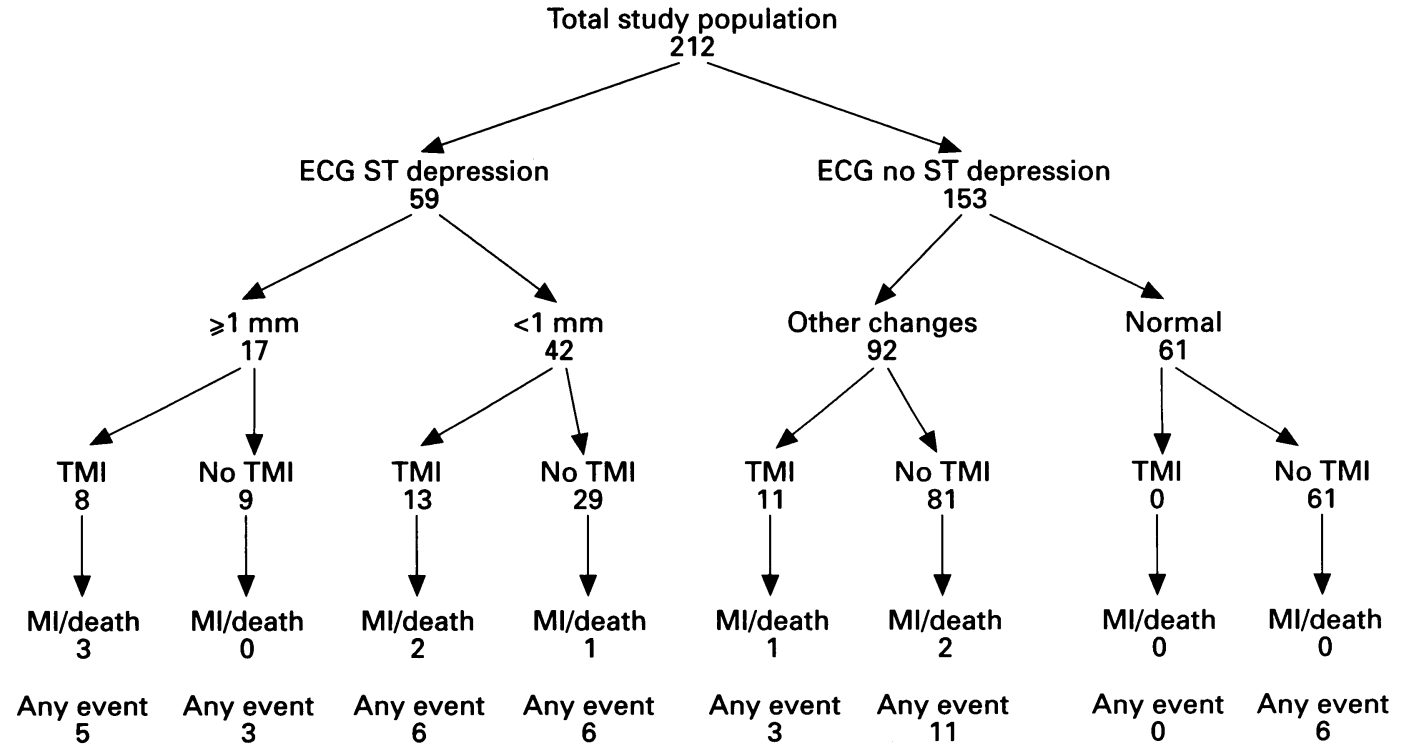


Table 4 Univariate predictors of adverse outcome among the clinical characteristics

\begin{tabular}{lll}
\hline Characteristic & OR $(95 \%$ CI & P value \\
\hline Age & $1.00(0.97$ to 1.04$)$ & 9.86 \\
Age > 65 years & $1.24(0.50$ to 1.98$)$ & 0.56 \\
Male sex & $1.25(0.51$ to 3.06$)$ & 0.63 \\
Smoking history & $1.25(0.48$ to 3.22$)$ & 0.65 \\
Family history & $1.44(0.70$ to 2.94$)$ & 0.32 \\
Diabetes & $0.70(0.15$ to 3.27$)$ & 0.65 \\
Hypertension & $0.90(0.43$ to 1.87$)$ & 0.78 \\
Previous stable angina. & $1.32(0.65$ to 2.68$)$ & 0.44 \\
Previous myocardial infarction & $0.76(0.37$ to 1.54$)$ & 0.44 \\
New onset angina & $0.72(0.36$ to 1.45$)$ & 0.36 \\
Maintenance BB Rx & $2.95(1.45$ to 5.98$)$ & 0.003 \\
Maintenance Ca. Ant. & $1.46(0.72$ to 2.96$)$ & 0.30 \\
Maintenance nitrates & $1.28(0.64$ to 2.57$)$ & 0.49 \\
Aspirin at admission & $0.82(0.40$ to 1.69$)$ & 0.60 \\
Heparin administered in the CCU & $1.32(0.65$ to 2.68$)$ & 0.44 \\
\hline
\end{tabular}

BB Rx, $\beta$ Block treatment, Ca. Ant., calcium antagonist; CCU, coronary care unit.

Table 5 Univariate predictors of adverse outcome from the admission ECG and continuous $S T$ segment monitoring

\begin{tabular}{llr}
\hline 12 lead ECG or ST monitoring variable & OR $(95 \% \mathrm{CI})$ & P value \\
\hline Normal & $0.375(0.15$ to 0.95$)$ & 0.038 \\
Any ST depression: & $3.41(1.67$ to 6.97$)$ & 0.001 \\
ST depression $\geqslant 0.1 \mathrm{mV}$ & $5.91(2.04$ to 17.10$)$ & 0.001 \\
ST depression <0.1 mV & $2.66(1.17$ to 6.03$)$ & 0.019 \\
Biphasic/inverted T wave & $1.29(0.65$ to 2.58$)$ & 0.46 \\
$\quad$ Biphasic/inverted T wave without ST & $0.75(0.35$ to 1.60$)$ & 0.45 \\
depression & $0.51(0.11$ to 2.33$)$ & 0.39 \\
ST elevation & $0.44(0.16$ to 1.20$)$ & 0.11 \\
Q waves & $1.44(0.15$ to 14.26$)$ & 0.75 \\
RBBB & $4.61(2.04$ to 10.38$)$ & $<0.001$ \\
Transient myocardial ischaemia &
\end{tabular}

RBBB, right bundle branch block.

predictive of adverse outcome $(P=0.003)$. Age, sex, risk factor profile, class of unstable angina as well as the use of heparin, aspirin, calcium antagonist, nitrate treatment, or a previous history of myocardial infarction were not predictive of outcome.

Among the resting electrocardiographic changes (table 5), a normal ECG at admission conferred a good prognosis $(P=0.038)$. ST segment depression regardless of severity was highly predictive of an unfavourable outcome $(P<0.001)$, while $T$ wave changes with or without ST depression and other ECG changes were not predictive of outcome. The presence of transient myocardial ischaemia during ST segment monitoring was the strongest predictor of adverse outcome (OR 4.61 (95\% CI 2.04 to 10.38$), P<0.001)$.

Multivariate stepwise logistic regression analysis showed the presence of transient myocardial ischaemia to be the strongest independent predictor of outcome (OR 2.94 (95\% CI $1 \cdot 14$ to $7 \cdot 54), P=0.025)$. Maintenance $\beta$ blocker treatment before admission also independently conferred an adverse prognosis (OR $2 \cdot 85$ (95\% CI 1.35 to 6.01$), P=0.01)$. ST depression on the resting 12 lead ECG showed a trend towards predicting an adverse prognosis in addition to the aforementioned two variables (OR $2.11(95 \%$ CI 0.93 to $4 \cdot 82), P=0 \cdot 076)$.

\section{Discussion}

This study has shown that transient episodes of ST segment depression during continuous monitoring are a powerful predictor of adverse outcome and have independent prognostic value beyond the clinical and ECG characteristics at presentation. The results also demon- strate that a $0 \cdot 1 \mathrm{mV}$ cut off for ST segment depression on a resting ECG is arbitrary and lesser degrees of ST segment depression still retain useful predictive value. Conversely, $T$ wave changes whether present in isolation or associated with ST segment changes have no such predictive value. In addition, patients with a normal ECG at presentation have a favourable outcome with a low frequency of subsequent transient ischaemia. The results of this study also show that patients developing unstable angina who are already receiving $\beta$ blocker treatment are more likely to have refractory symptoms requiring emergency revascularisation.

\section{ST SEGMENT MONITORING}

The presence of transient myocardial ischaemia during continuous ST segment monitoring has been shown in previous studies to predict adverse prognosis, ${ }^{73-16}$ however, this investigation has usually been performed in preselected patients with ST segment depression of $\geqslant 0.1 \mathrm{mV}$ on a resting ECG. ${ }^{14}{ }^{15}$ In a study of unselected patients with unstable angina, Wilcox et $a l^{17}$ found continuous ST segment monitoring to be less sensitive, but more specific, than the development of recurrent chest pain as a predictor of adverse outcome. In another report, Langer et $a l^{7}$ found ST segment monitoring to be highly predictive of an unfavourable outcome in patients with a sudden exacerbation of stable anginal symptoms. Langer also showed that ST segment depression of $\geqslant 0.1 \mathrm{mV}$ on serial 12 lead ECGs was also predictive of adverse outcome. However, this was less common, and not independently predictive of the findings of continuous ST segment monitoring. The ECG data described in their report were based on the selection of the "worst" ECG from a series of ECGs performed during hospitalisation and not solely on the admission ECG. This selection process in effect identifies only the most overt examples of ST shift, which would have been detected by ST segment monitoring in any case, and may explain in part the discrepancy between our reports. The greater frequency of ischaemic activity identified by continuous ST segment monitoring than by serial ECG recordings is likely to be related to silent episodes of ischaemia and brief ischaemic episodes where a resting ECG would be difficult to record. In addition, some of the differences could also be explained by different patient populations as a result of different inclusion criteria. We have also shown that patients with minor ST segment depression on the resting ECG had fewer events and less transient ischaemic activity than those with more pronounced depression.

One further reason for the reduced frequency of ischaemia in this study may have been the degree of standardisation of drug treatment achieved during ST segment monitoring. This reflects current management of such patients and, as has been previously reported, ${ }^{19}$ demonstrates the effectiveness of maximal antianginal medication in reducing myocardial ischaemia. Persistence of either 
silent or symptomatic transient myocardial ischaemia despite maximal treatment augers for a bad prognosis.

RESTING ECG

The prognostic importance of ST segment depression on a resting ECG shown in this study is consistent with that in previous published reports. ${ }^{12} 4$ 7-9 The results reported here demonstrate that a $0.1 \mathrm{mV}$ cut off for ST segment depression is arbitrary with minor degrees of ST segment depression still retaining useful predictive value. The likelihood of detecting such changes will depend on their duration and severity, and therefore ultimately on the frequency and timing of ECG recordings relative to symptoms. Only 17 patients had $\geqslant 1$ ST segment depression of whom three patients (fig 2) died or had a myocardial infarction. All these patients also had transient ST segment shift during continuous monitoring. Of the remaining 195 patients, six further patients died or had a myocardial infarction, three of these remaining patients having transient ischaemia. Thus continuous ST segment monitoring seems to have greater sensitivity in detecting high risk subgroups than $1 \mathrm{~mm}$ ST segment depression on the admission ECG. When minor ST segment depression on the admission ECG is also included the sensitivity of detecting high risk patients is similar to that of the presence of transient ischaemia and when combined are able to identify most patients at increased risk of adverse outcome.

$T$ wave changes on the resting ECG, mostly devoid of associated ST segment changes and lacking in any prognostic value, were present in 95 patients. While previous studies have reported $\mathrm{T}$ wave changes, ${ }^{10-12} \mathrm{ST}$ segment changes have not been classified separately. Although anterior $T$ wave inversion has been shown to predict a high likelihood of a lesion in the left anterior descending artery and an adverse prognosis, it was relatively uncommon in these reports in the absence of non- $Q$ wave myocardial infarction. Furthermore, these studies did not assess the frequency and prognostic significance of lesions in the left anterior descending artery in patients without $T$ wave inversion. ${ }^{10} \mathrm{~T}$ wave changes though common were associated with less transient ischaemia and their aetiology in this study was not that of acute myocardial infarction as these were specifically excluded by serial cardiac enzymes. As $\mathrm{T}$ wave changes can be caused not only by acute ischaemia but also by left ventricular hypertrophy and previous non- $Q$ wave myocardial infarction, patients may show greater pathological heterogeneity. The presence of $T$ waves may bias the physician towards hospitalising patients presenting with chest pain, even though they may not be a manifestation of acute ischaemia.

\section{PREADMISSION DRUG TREATMENT}

The increased morbidity of patients admitted with unstable angina and receiving $\beta$ blocker treatment is at first surprising, but may simply reflect more extensive underlying coronary artery disease, as the indication for $\beta$ blockers in many of these patients was pre-existing stable angina. Furthermore, patients presenting with anginal pain while receiving $\beta$ blockers are by definition demonstrating failure of antianginal treatment and are thus less likely to be stabilised by additional antianginal drugs. This was supported to some extent by the presence of more prolonged ischaemia in these patients than in those admitted without $\beta$ blockers. Although the combination of nitrates or calcium antagonists with $\beta$ blockers has been shown to reduce anginal frequency and transient ischaemia in unstable angina, no study has shown an improvement in outcome. Our findings are supported by the Holland Interuniversity Nifedipine Metoprolol Trial in which event rates in admitted patients receiving $\beta$ blockers were higher than in those started with $\beta$ blockers after admission, although statistical significance was not tested for this comparison. ${ }^{20}$ In another study reported by Muller et $a l,{ }^{21}$ patients receiving maintenance $\beta$ blockers were also less likely to remain symptom free than those given $\beta$ blockers after admission, although again this comparison was not tested for significance. Patients admitted with unstable angina while receiving $\beta$ blockers did not have a significant increase in myocardial infarction or death. Our results, however, show that these patients are more likely to continue with prolonged ischaemia and less well controlled symptoms necessitating emergency revascularisation and should therefore be considered for early diagnostic angiography.

\section{LIMITATIONS}

Adverse outcome in this study included emergency revascularisation which could be considered a subjective end point. Patients with refractory chest pain are, however, well recognised as representing a high risk subgroup for the development of myocardial infarction or death, ${ }^{1}$ and we only considered an adverse outcome if patients were transferred to a tertiary cardiothoracic centre during the index hospitalisation and underwent revascularisation as an emergency. In addition, despite the low rate of myocardial infarction and death, transient myocardial ischaemia and ST segment depression on the resting ECG remained significant predictors for this more objective end point.

Although we did not incorporate coronary angiography or any assessment of left ventricular function in the study, our results indicate a method of risk stratification to allow selection of patients for such investigations at an early stage, whereas patients at low risk could be managed medically in the first instance.

\section{CONCLUSIONS}

The presence of transient ST segment shift during continuous ST segment monitoring is a powerful predictor of adverse prognosis in patients with unstable angina over and above the clinical and ECG characteristics on admission. ST segment depression on the admission ECG even if $<0.1 \mathrm{mV}$ is predictive of adverse outcome, while $\mathrm{T}$ wave changes, although 
common, have no such prognostic value. Patients presenting with a normal ECG have a good prognosis and a low probability of subsequent transient ischaemia and those presenting while receiving maintenance $\beta$ blockers are more likely to have refractory symptoms unresponsive to further medical treatment.

Continuous ST segment monitoring should be performed more frequently in patients with unstable angina as simple clinical criteria combined with ST segment monitoring are an effective method of identifying high risk subsets in the early phase after admission.

We would like to thank the coronary care staff at the Royal United Bath, Hillingdon, and High Wycombe hospitals whose support was invaluable in the completion of the study, and Ms D Clarke for technical assistance and preparing the figures. DJP has been supported by a fellowship from the Augustus Newman Foundation and CJK by a junior fellowship from the British Heart Foundation.

1 Gazes PC, Mobley EM, Faris HM, Duncan RC Humphries GB. Preinfarctional (unstable) angina-a
prospective study-ten year follow-up. Circulation 1973; 58:331-7.

2 Cairns JA, Gent MG, Singer J, et al. Aspirin, sulphinpyrazone, or both in unstable angina. $N$ Engl f Med 1985; 313:1369-75.

3 Mulcahy $\mathrm{R}$, Al Awadhi AH, de Buitleor $M$, Tobin $R$, Johnson $H$, Contoy $R$. Natural history and prognosis of unstable angina. Am Heart $\mathcal{f}$ 1985;109:753-8.

4 The RISC Group. Risk of myocardial infarction and death during treatment with low dose aspirin and intravenous heparin in men with unstable coronary artery disease. heparin in men with un

5 Karlson BW, Herlitz J, Pettersson P, Hallgren P, Strombom U, Hjalmarson A. One-year prognosis in patients hospitalised with a history of unstable angina pectoris. Clin Cardiol 1993;16:397-402.

6 Theroux P, Waters D, Qiu S, McCans J, de Guise P Juneau $M$. Aspirin versus heparin to prevent myocardial infarction during the acute phase of unstable angina. Circulation 1993;88:2045-8.

7 Langer AL, Freeman MR, Armstrong PW. ST segment shift in unstable angina: pathophysiology and association with coronary anatomy and hospital outcome. $₹ \mathrm{Am} \mathrm{Coll}$ Cardiol 1989;13:1495-502.

8 Cohen M, Hawkins L, Greenberg S, Fuster V. Usefulness of ST-segment changes in $\geqslant 2$ leads on the emergency room electrocardiogram in either unstable angina or nonQ-wave myocardial infarction in predicting outcome. $\mathrm{Am}$ f Cardiol 1991;67:1368-73.

9 Severi S, Orsini E, Marraccini P, et al. The basal electrocardiogram and the exercise stress test in assessing prognosis in patients with unstable angina. Eur Heart $\mathcal{f}$ 1988;9: $441-6$.

10 de Zwann C, Bar FW, Wellens HJ. Characteristic electrocardiographic pattern indicating a critical stenosis high in left anterior descending coronary artery in patients admitted because of impending myocardial infarction. Am Heart $\mathcal{F} 1982 ; 103: 730-5$.

11 Haines DE, Raabe DS, Gundel WD, Wackers FJ. Anatomic and prognostic significance of new T-wave inversion in unstable angina. Am $\mathcal{F}$ Cardiol 1983;52:14-18.

12 Sclarovsky S, Rechavia E, Strasberg B, et al. Unstable angina: ST segment depression with positive versus negative $T$ wave deflections-clinical course, ECG evolution, and angiographic correlation. Am Heart $\mathcal{f}$ 1988;116: and angioe 41 .

13 Johnson S, Mauritson DR, Winniford MD, et al. Continuous electrocardiographic monitoring in patients
with unstable angina pectoris: identification of high-risk with unstable angina pectoris: identification of high-risk subgroup with severe coronary disease, variant angina,
and/or impaired early prognosis. Am Heart $\mathcal{f} 1982 ; 103$ : 4-12.

14 Nademanee $K$, Intarachot V, Josephson MA, Rieders D, Mody FV, Singh BN. Prognostic significance of silent myocardial ischemia in patients with unstable angina. $\mathcal{F}$ Am Coll Cardiol 1987;10:1-9.

15 Gottlieb SO, Weisfeldt ML, Ouyang P, Mellits ED, Gerstenblith G. Silent ischemia as a marker for early unfavourable outcomes in patients with unstable angina. N Engl ₹ Med 1986;314:1214-9.

16 Von Armin TH, Gerbig HW, Krawietz W, Hofling B. Prognostic implications of transient-predominantly silent-ischaemia in patients with unstable angina silent-ischaemia in patients with
pectoris. Eur Heart $\mathcal{f} 1988 ; 9: 435-40$.

17 Wilcox I, Freedman SB, Kelly DT, Harris P. Clinical significance of silent ischemia in unstable angina pectoris. nificance of silent ischemia in
Am $\mathcal{F}$ Cardiol 1990;65:1313-6.

18 Holdright D, Patel D, Cunningham D, et al. Comparison of the effects of heparin and aspirin versus aspirin alone on transient myocardial ischemia and in-hospital prognosis in patients with unstable angina. $\mathcal{f}$ Am Coll Cardiol 1994;24:39-45.

19 Grambow DW, Topol EJ. Effect of maximal medical therapy on refractoriness of unstable angina pectoris. $\mathrm{Am} \mathcal{F}$ Cardiol 1992;70:577-81.

20 HINT Research Group. Early treatment of unstable angina in the coronary care unit: a randomised double blind placebo controlled comparison of recurrent ischaemia in patients treated with nifedipine or metoprolol or both. $\mathrm{Br}$ Heart $₹$ 1986;56:400-13.

21 Muller JE, Zoltan GT, Pearle DL, et al. Nifedipine and conventional therapy for unstable angina: a randomised, double-blind comparison. Circulation 1984;69:728-39. 